\title{
Neutrino oscillations in a magnetic field: the three-flavor case
}

\author{
Alexey Lichkunov ${ }^{* a}$, Artem Popov ${ }^{a}$, Alexander Studenikin ${ }^{a b}$ \\ ${ }^{a}$ Department of Theoretical Physics, Lomonosov Moscow State University \\ 119991 Moscow, Russia \\ ${ }^{b}$ Joint Institute for Nuclear Research \\ Dubna 141980, Moscow Region, Russia \\ E-mail: ar.popov@physics.msu.ru, studenikesrd.sinp.msu.ru
}

\begin{abstract}
We adopt the approach to the problem of neutrino oscillations in a magnetic field introduced in [1] and extended to the case of three neutrino generations in [2] to investigate the impact of ultrahighenergy neutrino oscillations in the interstellar magnetic field. Based on the assumption that UHE neutrinos flavour distribution follows the pattern 1:2:0, we have estimated the fluxes detected by a terrestrial neutrino telescope. We find out that the interaction with a magnetic field does not significantly modify the flavour distribution of UHE neutrinos unless they possess transition magnetic moments.
\end{abstract}

40th International Conference on High Energy physics - ICHEP2020

July 28 - August 6, 2020

Prague, Czech Republic (virtual meeting)

\footnotetext{
* Speaker.
} 


\section{Ultrahigh-energy neutrino oscillations in a magnetic field}

In [2] we showed that the probabilities of neutrino spin-flavour oscillations in a magnetic field can be expressed by the following decomposition

$$
\begin{aligned}
P\left(v_{\alpha}^{h} \rightarrow v_{\beta}^{h^{\prime}} ; x\right) & =\delta_{\alpha \beta} \delta_{h h^{\prime}}-4 \sum_{\{i, j, s, \sigma\}} \operatorname{Re}\left(\left[A_{\alpha \beta}^{h h^{\prime}}\right]_{i, j, s, \sigma}\right) \sin ^{2}\left(\omega_{i j}^{s s^{\prime}} x / 2\right) \\
& +2 \sum_{\{i, j, s, \sigma\}} \operatorname{Im}\left(\left[A_{\alpha \beta}^{h h^{\prime}}\right]_{i, j, s, \sigma}\right) \sin \left(\omega_{i j}^{s s^{\prime}} x\right),
\end{aligned}
$$

where the amplitude coefficients were introduced

$$
\left[A_{\alpha \beta}^{h h^{\prime}}\right]_{i, j, s, \sigma}=U_{\beta i}^{*} U_{\alpha i} U_{\beta j} U_{\alpha j}^{*} C_{i s}^{h^{\prime} h}\left(C_{j \sigma}^{h^{\prime} h}\right)^{*},
$$

and

$$
\sum_{\{i, j, s, \sigma\}}=\sum_{i>j ; s, \sigma}+\sum_{s>\sigma ; i=j}
$$

where $\alpha, \beta$ are neutrino flavours $e, \mu, \tau$ and $h, h^{\prime}$ are neutrino helicities. In general, the probabilities of neutrino oscillations exhibit a complicated interplay of oscillations on both vacuum $\Delta m_{i j}^{2} / 4 p$ and magnetic $\mu_{v} B$ frequencies [1]. Antineutrino oscillations probabilities $\bar{P}\left(v_{\alpha}^{h} \rightarrow v_{\beta}^{h^{\prime}} ; x\right)$ can be obtained by replacing $U \rightarrow U^{*}$ or, equivalently, $x \rightarrow-x$. Fig. 1 shows the probabilities of $v_{e} \rightarrow v_{e}$ ,$v_{e} \rightarrow v_{\mu}$ and $v_{e} \rightarrow v_{\tau}$ oscillations as functions of distance in parsec for the case of neutrino energy equal to $10 \mathrm{ZeV}$. Below we connect the oscillations probabilities (1) with the observable neutrino fluxes.

Astrophysical objects as active galactic nuclei, gamma ray bursts and supernova remnants are considered to be the sources of ultrahigh-energy (UHE) neutrinos, i.e. neutrinos with energies higher that $1 \mathrm{PeV}$. It is expected that UHE neutrinos flavour distribution follows a simple pattern $\Phi_{e}^{0}: \Phi_{\mu}^{0}: \Phi_{\tau}^{0}=1: 2: 0$. In [3] the impact of neutrino interaction with the interstellar magnetic field on neutrino oscillations was analysed in the two-flavour approximation. In this paper we consider neutrino oscillations in the interstellar magnetic field in the three flavour case. Given the initial neutrino fluxes $\Phi_{\alpha}^{0}$, the fluxes at the distance $x$ are computed as follows

$$
\Phi_{\alpha}(x)=\sum_{\beta} \Phi_{\beta}^{0} P_{\alpha \beta}(x)
$$

where $P_{\alpha \beta}(x)$ are the active-active transitions probabilities $P\left(v_{\alpha}^{L} \rightarrow v_{\beta}^{L}\right)$. As neutrinos travel throughout cosmic scales, they undergo the wave packet separation resulting in the decoherence effect. Thus, to calculate the fluxes at a neutrino telescope we replace $P_{\alpha \beta}(x)$ in eq. (4) with the distanceaveraged probabilities $\left\langle P_{\alpha \beta}\right\rangle$, which can be obtained from eq. (1):

$$
\left\langle P_{\alpha \beta}\right\rangle=\delta_{\alpha \beta}-2 \sum_{\{i, j, s, \sigma\}} \operatorname{Re}\left(\left[A_{\alpha \beta}^{L L}\right]_{i, j, s, \sigma}\right) .
$$

Note that $\left\langle\bar{P}_{\alpha \beta}\right\rangle=\left\langle P_{\alpha \beta}\right\rangle$ since the distance-odd parts of the probabilities disappear after averaging.

It is known that after vacuum oscillations and decoherence, neutrino distribution by flavours is almost uniform: $\Phi_{e}: \Phi_{\mu}: \Phi_{\tau} \approx 1: 1: 1$. Using (5), we find out that the pattern 1:1:1 holds 
even if we consider oscillations of Dirac neutrinos with non-zero diagonal magnetic moments in the interstellar magnetic field. Since $\left\langle\bar{P}_{\alpha \beta}\right\rangle=\left\langle P_{\alpha \beta}\right\rangle$, antineutrino flavour distribution follows the same relation $\bar{\Phi}_{e}: \bar{\Phi}_{\mu}: \bar{\Phi}_{\tau} \approx 1: 1: 1$.

However, this is the case only if neutrinos do not possess transition magnetic moments. Oscillations of neutrinos with non-diagonal magnetic moments (particularly Majorana neutrinos) require more thorough analysis.

\section{Acknowledgements}

The work is supported by the Russian Foundation for Basic Research under grant No. 2052-53022-GFEN-a. The work of A.P. is supported by the Foundation for the Advancement of Theoretical Physics and Mathematics "BASIS" under grant No. 19-2-6-209-1

\section{References}

[1] A. Popov and A. Studenikin, Eur. Phys. J. C 79 (2019) no.2, 144 doi:10.1140/epjc/s10052-019-6657-z [http://arXiv:1902.08195 [hep-ph]].

[2] A. Lichkunov, A. Popov and A. Studenikin, Neutrino eigenstates and oscillations in a magnetic field, POS EPS-HEP 2019 (2020) 415.

[3] P. Kurashvili, K. Kouzakov, L. Chotorlishvili and A. Studenikin, Spin-flavor oscillations of ultrahigh-energy cosmic neutrinos in interstellar space: The role of neutrino magnetic moments, Phys. Rev. D 96 (2017) 103017, arXiv:1711.04303 [hep-ph].

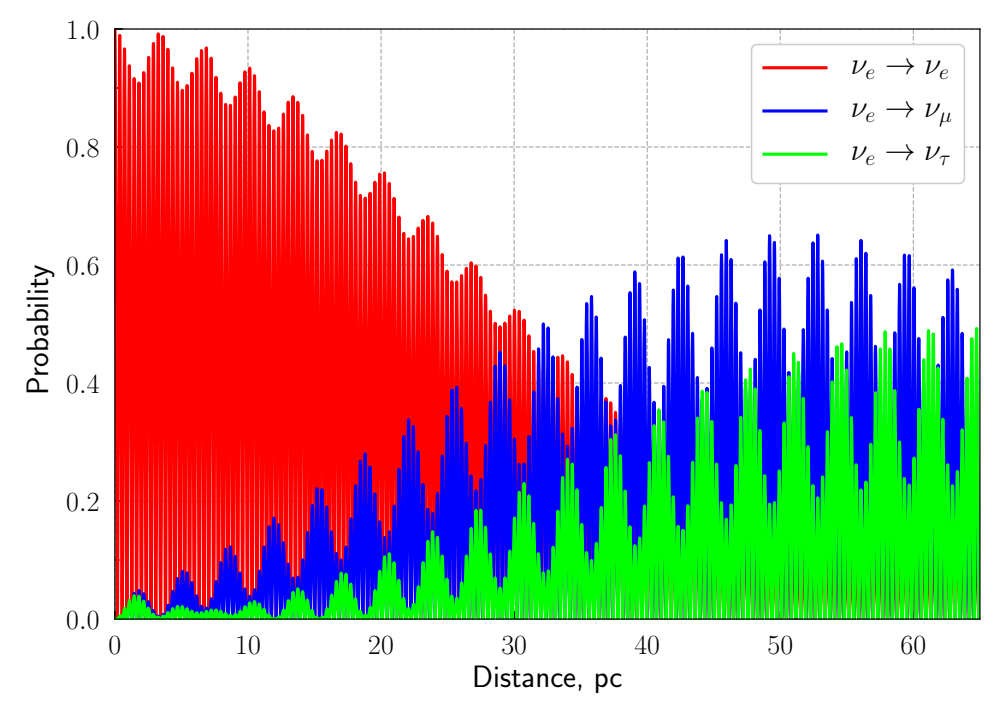

Figure 1: The probabilities of $v_{e} \rightarrow v_{e}, v_{e} \rightarrow v_{\mu}$ and $v_{e} \rightarrow v_{\tau}$ oscillations as functions of distance in parsec for the case of neutrino energy $10 \mathrm{ZeV}$. 